\title{
Desregulación del eje hipotalámico-pituitario-adrenal iniciada por un patrón binge drinking, pero no por el consumo agudo de alcohol, en mujeres y hombres adolescentes
}

\author{
Hypothalamic-pituitary-adrenal axis dysregulation \\ initiated by a binge drinking pattern, but not by acute \\ alcohol intake, in female and male adolescents
}

\author{
Milton Ramírez-Piña*, Santiago Monleón*, Concepción Vinader-Caerols*. \\ * Departamento de Psicobiología, Universitat de València, E-46010, Valencia.
}

\section{Resumen}

El consumo excesivo de alcohol es un problema de salud pública mundial, siendo los adolescentes y jóvenes adultos la población más afectada. El objetivo de este estudio fue esclarecer los efectos de una historia binge drinking (BD) y/o del consumo agudo de alcohol sobre la respuesta de estrés en mujeres y hombres. Participaron 235 adolescentes (143 mujeres y 92 hombres). Se evaluaron cortisol, presión arterial sistólica y diastólica (PAS y PAD), frecuencia cardiaca (FC) y estrés percibido (EP). En el experimento 1, los efectos del alcohol fueron estudiados separadamente en mujeres y hombres debido a diferencias en la concentración de alcohol en sangre (CAS). En el experimento 2, una selección balanceada de mujeres y hombres con similar CAS permitió su comparación. En el experimento 1, las mujeres que recibieron alcohol mostraron un incremento de cortisol y FC, y las binge drinkers mostraron mayor FC que las abstemias. Los hombres abstemios que recibieron alcohol mostraron mayor FC y los binge drinkers tuvieron niveles más altos de cortisol y PAS que los abstemios. En el experimento 2, se observaron resultados similares y diferencias de sexo, mostrando los hombres niveles más altos de cortisol y PAS, y menos EP que las mujeres. En conclusión, la respuesta normal del eje HPA adolescente al consumo de alcohol refleja un incremento de cortisol en mujeres, así como de FC en ambos sexos. Además, una historia de consumo BD está asociada con una desregulación del eje HPA, manifestado con niveles más altos de cortisol (independientemente de sexo), PAS en varones y FC en mujeres, adolescentes sanos

Palabras clave: Consumo intensivo de alcohol; alcohol; respuesta de estrés; eje hipotalámico-pituitario-adrenal; adolescentes.

\section{Abstract}

Excessive alcohol consumption is a worldwide public health problem, being adolescents and young adults the population most affected by this problem. The aim of this study was to clarify the effects of having a history of binge drinking (BD) and/or acute alcohol consumption on the stress response in female and male adolescents. Participants were 235 adolescents (143 females and 92 males). Cortisol, systolic and diastolic blood pressure (SBP and DBP), heart rate (HR) and perceived stress (PS) were evaluated in adolescents with different and similar blood alcohol concentrations (BAC). In Experiment 1, the effects of alcohol were studied separately in females and males because of differences in BAC. In Experiment 2, a direct comparison between sexes was carried out in a counterbalanced selection of participants with similar BAC. In Experiment 1, females receiving alcohol showed an increase in cortisol and HR, and binge drinkers displayed higher HR than refrainers. Male refrainers receiving alcohol showed higher HR, and binge drinkers showed higher cortisol and SBP than refrainers. In Experiment 2, similar results were observed and sex differences were evident, with males showing higher cortisol and SBP, and lower PS than females. In conclusion, the normal response of the adolescent HPA axis to alcohol consumption is an increase in cortisol levels in females, as well as HR in both sexes. In addition, a history of BD is associated with HPA axis dysregulation, which is manifested by higher values of cortisol (independently of sex), SBP in male and HR in female healthy adolescents.

Key words: Binge drinking; alcohol; stress response; adrenal-pituitaryhypothalamic axis; adolescents.

Recibido: Febrero 2021; Aceptado: Septiembre 2021.

Enviar correspondencia a: Prof. Dr. Concepción Vinader-Caerols. Departamento de Psicobiología, Facultad de Psicología, Universitat de València. Blasco Ibáñez, 21, 46010 Valencia, España. Tfno. 9638646 52. Fax 963864668.

E-mail: concepcion.vinader@uv.es 
$\mathrm{E}$ 1 consumo excesivo de alcohol es un problema mundial de salud pública y los adolescentes y adultos jóvenes son los más afectados en España y en otros países. La mayoría de esta población desarrolla un patrón de consumo conocido como consumo intensivo de alcohol o binge drinking (BD), definido por el National Institute on Alcohol Abuse and Alcoholism (NIAAA, 2004) como un patrón de consumo en el que la concentración de alcohol en sangre (CAS) aumenta a 0,8 g/L o más. El BD se caracteriza por la ingesta de grandes cantidades de alcohol en un corto período de tiempo (dos horas), seguido de un período de abstinencia que puede variar entre una semana y un mes (Parada et al., 2011; Vinader-Caerols y Monleón, 2019). La prevalencia de BD en España es alta: el 32,3\% de los estudiantes españoles entre 14 y 18 años de edad admitieron en una reciente encuesta haber participado en este tipo de consumo en los últimos 30 días (Observatorio Español de las Drogas y las Adicciones, 2020). Este patrón se asocia comúnmente con un deterioro agudo de la coordinación motora y el funcionamiento cognitivo, y su continuación durante la adolescencia predice una respuesta atípica al estrés del cortisol en la edad adulta joven (Hagan et al., 2019).

Aunque el alcohol a menudo se consume para aliviar el estrés (Keyes, Hatzenbuehler, Grant y Hasin, 2012), activa algunos sistemas cerebrales de estrés pudiendo ser considerado un estresor en sí mismo (Becker, 2017). El estudio de las interacciones entre los sistemas de estrés biológicos y el consumo de alcohol se ha centrado en el eje hipotalámico-pituitario-adrenal (HPA) (Weera y Gilpin, 2019) y en la principal hormona de estrés, el cortisol (Hellhammer y Schubert, 2012), utilizado como marcador bioquímico en este tipo de investigaciones (González-Cabrera, Fernández-Prada, Iribar-Ibabe y Peinado, 2014). Teniendo esto en cuenta, son necesarios estudios que evalúen la disfunción del eje HPA, ya que este es el mediador principal de los efectos a corto plazo del alcohol en el cuerpo y una vía potencial mediante la cual el alcohol ejerce efectos a largo plazo en los sistemas biológicos (Hagan et al., 2019).

La creciente evidencia sugiere que el alcohol estimula directamente el eje HPA y tiene efectos sobre los receptores de glucocorticoides en los circuitos extrahipotalámicos del cerebro anterior límbico y de la corteza prefrontal medial que contribuyen al desarrollo de los trastornos por uso de alcohol (TUAs), a su progresión, cronicidad y riesgo de recaída (Blaine y Sinha, 2017). El efecto agudo del alcohol sobre las hormonas del estrés, estudiado a través del consumo voluntario de alcohol en humanos y animales, aumenta el cortisol y la corticosterona (King, Munisamy, de Wit y Lin, 2006; Lu y Richardson, 2014). En adolescentes, el consumo agudo de alcohol estimula el eje HPA, lo que resulta en un aumento inicial de la producción de cortisol (Magrys, Olmstead, Wynne-Edwards y Balodis, 2013). La literatura también muestra que el BD durante la adoles- cencia predice una respuesta al estrés atípica del cortisol durante la edad adulta joven: a corto plazo, los sujetos con una historia de BD muestran niveles basales de cortisol significativamente más altos, lo que indica una sobrerregulación del eje HPA (Wemm et al., 2013); por el contrario, a largo plazo, el BD durante la adolescencia se asocia con una respuesta al estrés del cortisol significativamente menor en adultos jóvenes (Hagan et al., 2019). Los estudios que evalúan la relación entre el consumo agudo de alcohol y la historia de BD han asociado las CAS por debajo de $0,8 \mathrm{~g} / \mathrm{L}$, en consumidores con una historia de consumo entre bajo y moderado (no binge drinkers), con una mayor respuesta del eje HPA (mayores niveles de cortisol en sangre); sin embargo, cuando los sujetos son binge drinkers se ha relacionado a estas CAS con una respuesta reducida del eje HPA (Allen, Lee, Koob y Rivier, 2011; Blaine y Sinha, 2017; Waltman, Blevins, Boyd y Wand, 1993; Zimmermann et al., 2004).

El cortisol también facilita un incremento de la actividad cardiovascular, elevando la frecuencia cardíaca y la presión arterial (Hagan et al., 2019; Ulrich-Lai y Herman, 2009). Varios estudios han demostrado que una ingesta aguda de alcohol (Bau et al., 2011; Sher, Bartholow, Peuser, Erickson y Wood, 2007; Vinader-Caerols, Monleón, Carrasco y Parra, 2012) o un patrón BD sostenido (King, Houle, de Wit, Holdstock y Schuster, 2002) aumenta la frecuencia cardíaca (FC) en adultos jóvenes. También se ha señalado que el BD frecuente durante la adolescencia puede aumentar el riesgo de desarrollar presión arterial (PA) elevada en adultos jóvenes, independientemente de otros factores de riesgo como tabaquismo u obesidad (Hayibor, Zhang y Duncan, 2019).

El estrés percibido se ha asociado con el riesgo de abuso de alcohol en adolescentes y adultos jóvenes (Tavolacci et al., 2013). En coherencia con esto, se ha encontrado que los estudiantes con un patrón marcado de consumo de alcohol BD puntúan casi el doble que la población adulta general en la escala de estrés percibido (Bidwal, Ip, Shah y Serino, 2015).

Los resultados experimentales de investigaciones realizadas con un solo sexo a veces son extrapolados a ambos. El sexo debe ser considerado como una variable biológica importante en la investigación básica y preclínica antes de aplicar los resultados a hombres y mujeres (Lee, 2018). Por tanto, decidimos comparar los sexos con respecto a los efectos del alcohol sobre el eje HPA según la historia de BD. Con este objetivo en mente, se diseñaron dos experimentos: el primero analizó a hombres y mujeres por separado con diferentes CAS; el segundo evaluó una selección de mujeres y hombres con CAS similares, lo que permitió estudiar el efecto del género.

Teniendo en cuenta las pruebas publicadas antes mencionadas, es necesario realizar un análisis sistemático de los efectos del consumo agudo de alcohol sobre el eje HPA y 
sobre la activación del sistema nervioso autónomo. Dichos estudios experimentales con población adolescente son escasos, por lo que este estudio tuvo como objetivo principal aclarar los efectos de una historia de BD y/o del consumo agudo de alcohol sobre la funcionalidad del eje HPA (cortisol) y otras variables de la respuesta al estrés (FC, PA y EP) en adolescentes de ambos sexos. Otro objetivo fue proporcionar más pruebas sobre los cambios provocados en el eje HPA y en la respuesta al estrés en adolescentes sanos por el BD cuando se inicia desde una edad temprana, específicamente en los niveles de cortisol, FC, PA y EP. Estos cambios son de gran importancia, ya que pueden contribuir a la transición del BD a la pérdida compulsiva de control sobre la ingesta de alcohol que se observa en los TUAs graves. Para los objetivos mencionados, se seleccionó una muestra de binge drinkers (con historia de $\mathrm{BD}$ ) y no binge drinkers (abstemios y consumidores ocasionales sin historia de BD) que recibieron una bebida de control o una bebida alcohólica como condiciones experimentales.

Recreamos las condiciones en las que el BD suele ocurrir -es decir, un consumo de riesgo de alcohol $(38,4 \mathrm{~g})$ en un corto tiempo (20 min) - y medimos la funcionalidad del eje HPA (niveles de cortisol). Esperamos ver un aumento en los niveles de cortisol, FC, PA y EP en los hombres y mujeres adolescentes sin historia de $\mathrm{BD}$ debido a la reacción natural de la respuesta al estrés tras una ingesta aguda de alcohol. Además, los participantes con una historia de BD solamente ( $\sin$ ingesta de alcohol aguda) tendrían niveles basales más elevados de las variables evaluadas debido a la sobrerregulación del eje HPA. Por último, de acuerdo con la literatura, supusimos que nuestros participantes con un año de historia de BD mostrarían valores más bajos (niveles de cortisol, FC, PA y EP) tras el consumo agudo de alcohol.

\section{Método}

\section{Participantes}

Participaron en el estudio 235 estudiantes adolescentes sanos de 18-19 años de edad (143 mujeres y 92 hombres) de la Universitat de València (España). Los voluntarios fueron reclutados mediante un autoinforme de hábitos de consumo de alcohol y salud general. Los participantes se clasificaron como abstemios si nunca antes habían consumido bebidas alcohólicas (abstemios puros) o habían consumido muy esporádicamente (consumidores ocasionales) (ver Tabla 1); o como consumidores de alcohol con patrón BD según los criterios NIAAA para España (López-Caneda et al., 2014) si habían bebido según los hábitos de BD autoinformados por los sujetos, seis o más UBEs (Unidad de Bebida Estándar) de destilados (contenido alcohólico $\geq$ $40 \%$ vol.) de forma consecutiva en el caso de los hombres y cinco o más UBEs de forma consecutiva en el caso de las mujeres, un mínimo de tres veces al mes durante los últimos 12 meses (Vinader-Caerols y Monleón, 2019).

Se aplicaron estrictamente los siguientes criterios de inclusión/exclusión durante la selección de la muestra. Criterios de inclusión: edad entre 18-19 años; índice de masa corporal saludable (media de 22,88 \pm 0,30 en hombres; 21,44 \pm 0,23 en mujeres) y buena salud (reportando un estado de bienestar emocional y físico, sin problemas médicos importantes ni patología diagnosticada). Criterios de exclusión: toma de medicación; antecedentes de trastornos mentales (diagnosticados por un profesional sanitario según los criterios del DSM); patrón irregular de sueño (sueño no reparador y/u horario irregular); haber consumido, aunque sea esporádicamente, cualquier droga (aparte de alcohol o tabaco) o tener antecedentes de abuso de sustancias, incluyendo cafeína (nuestro criterio: $\leq 2$ bebidas estimulantes/día), tabaco (nuestro criterio: $\leq 10$ cigarrillos/día) o alcohol; haber sufrido un evento estresante intenso en el año previo a la experimentación y tener familiares de primer grado con antecedentes de alcoholismo. Se realizó una entrevista telefónica de aproximadamente 15 minutos con cada sujeto para confirmar la información proporcionada en el autoinforme y concertar la fecha y hora de la prueba. Se les dijo a los participantes que siguieran su patrón de sueño normal y su rutina habitual de comidas, y que almorzaran una hora antes de la sesión experimental.

Los datos del ciclo menstrual de las participantes se registraron en el autoinforme y en la entrevista telefónica. En la prueba se consideró la fase del ciclo de cada una para contrabalancear esta variable en cada grupo, comprobando que el número de mujeres en cada fase del ciclo fuese similar en cada grupo. No se incluyó en el estudio a mujeres que tomaban anticonceptivos.

\section{Pruebas y aparatos}

La actividad del eje HPA se midió analizando los niveles de cortisol en saliva utilizando una prueba de radioinmunoensayo competitivo de fase sólida. El Salivette ${ }^{\circledR}$ fue utilizado como método higiénico de recolección de saliva por medio de un hisopo sintético especialmente diseñado para la determinación de cortisol. Se recolectaron tres muestras de saliva a todos nuestros sujetos: una antes del consumo (COR0'), una segunda 20 min (COR20') después de la ingesta de la bebida y una tercera 50 min (COR50') después de la ingesta, considerando que los niveles altos de la CAS se observan en el intervalo de 20-50 min (Vinader-Caerols, Talk, Montañés, Duque y Monleón, 2017a; Vinader-Caerols, Duque, Montañés y Monleón 2017b). Las muestras se congelaron a $-18^{\circ} \mathrm{C}$ hasta que se enviaron al laboratorio para su análisis mediante un radioinmunoensayo competitivo en fase sólida (tubo recubierto) con el kit comercial Coat-A-Count C (DPC, Siemens Medical Solutions Diagnostics, Los Angeles, CA, EE. UU.). La sensibilidad del 
ensayo fue de $0,5 \mathrm{ng} / \mathrm{mL}(1,38 \mathrm{nmol} / \mathrm{L})$. Los datos se expresaron en unidades nanomolares $(\mathrm{nmol} / \mathrm{L})$. Todas las muestras de cada participante se analizaron en el mismo ensayo; los coeficientes de variación intra e interensayo fueron todos inferiores a 5,5\%. Los niveles de cortisol en saliva se determinaron en el Laboratorio de Análisis Echevarne en Valencia (España).

Se aplicó el Cuestionario de Identificación de los Trastornos debidos al Consumo de Alcohol (AUDIT) (Saunders, Aasland, Babor, de La Fuente y Grant, 1993) para medir el uso problemático de alcohol entre los sujetos. El AUDIT consta de 10 preguntas que evalúan la cantidad y frecuencia de la ingesta de alcohol y las conductas relacionadas con el alcohol y sus consecuencias. Utiliza un rango de 0 a 40 puntos, en el que una puntuación de 8 o más indica un uso problemático del alcohol. Una puntuación superior se relaciona con una mayor gravedad de la ingesta problemática de alcohol.

Se utilizó un tensiómetro digital automático (M10-IT, OMRON, España) para medir la PA sistólica (PAS), la PA diastólica (PAD) y la FC en todos los sujetos.

Los participantes fueron evaluados mediante la Escala de Estrés Percibido (PSS-14) (Cohen, Kamarck y Mermelstein, 1983), un cuestionario de autoinforme estandarizado de 14 ítems diseñado para medir el estrés y evaluar cómo de impredecible, incontrolable y sobrecargada consideran los encuestados que ha sido su vida durante el mes anterior. Una puntuación directa más alta en el PSS-14 indica un nivel más alto de EP. Todos los sujetos completaron la versión estandarizada en español de la PSS-14. La consistencia interna de esta escala se calculó para nuestros datos, obteniendo un coeficiente alfa de Cronbach de ,836 para mujeres y ,860 para hombres en el Experimento 1 y de ,861 en el Experimento 2 (mujeres y hombres juntos).

Se empleó un alcoholímetro (Alcoquant ${ }^{\circledR}$ 6020, Envitec, Alemania) para medir la CAS de cada participante antes y después (20 min y $50 \mathrm{~min}$ ) de la ingesta de una bebida (bebida de control o bebida alcohólica).

\section{Procedimiento}

El procedimiento experimental fue aprobado por el Comité de Ética en Investigación de la Universitat de València (número de certificación: H1485172642673; aprobado el 7 de julio de 2017) y era conforme a la Declaración de Helsinki. Todos los participantes dieron su consentimiento informado por escrito para participar en el estudio. De acuerdo con su patrón de consumo (abstemios y binge drinkers) y la bebida recibida (bebida de control o bebida alcohólica), los sujetos fueron asignados a una de cuatro condiciones experimentales: Abstemios-Control (A-Co) (abstemios puros-ingesta de bebida de control); Abstemios-Alcohol (A-A) (consumidores ocasionales-ingesta de bebida alcohólica); Binge Drinkers-Control (BD-Co) (sujetos con antecedentes de BD-ingesta de bebida de control) o Binge Drinkers-Alcohol (BD-A) (sujetos con antecedentes de BD-ingesta de bebida alcohólica).

Se les indicó a los participantes que se abstuvieran de consumir alcohol, bebidas con cafeína, drogas o medicación y de realizar ejercicio intenso durante las 24 horas anteriores a la sesión experimental, y que se abstuvieran de comer y fumar al menos 1 hora antes de la sesión. Al comienzo de la sesión, se midió la CAS en todos los sujetos utilizando el alcoholímetro para comprobar que no habían consumido alcohol. Además, se evaluó el uso problemático de alcohol entre los sujetos BD mediante la prueba AUDIT (puntuación media: 6,72 \pm 0,33 en mujeres y 7,69 \pm 0,47 en hombres). Ninguno de los sujetos resultó ser dependiente del alcohol. A todos los participantes se les tomó una primera muestra de saliva para la determinación de cortisol (COR0') justo antes de la ingesta. Cada sujeto recibió un refresco aromatizado (lima, naranja o cola, sin cafeína) envasado en latas de $330 \mathrm{ml}$, solo o mezclado (según el grupo experimental) con destilados con una graduación alcohólica de $40 \%$ vol. (vodka o ginebra) en dosis de riesgo de $120 \mathrm{ml}$ (equivalente a 38,4 g de alcohol). Los sujetos fueron instruidos a consumir su bebida en un plazo de 20 min, durante el cual comieron un aperitivo (el mismo para todos los participantes) y las bebidas siempre se consumieron en presencia de un ayudante de investigación. Después de terminar la bebida, todos los sujetos se enjuagaron la boca con agua y se sometieron a un período de espera de 20 minutos.

Tras este intervalo de espera de 20 min, se recogió la segunda muestra de saliva para la determinación de cortisol (COR20') y se midió la CAS en todos los sujetos. Posteriormente, medimos su PAS, PAD y FC (todas fueron registradas 3 veces y se calculó su promedio) y su EP mediante el PSS-14. Por último, los participantes proporcionaron una tercera muestra de saliva (COR50'), después de lo cual se midió nuevamente la CAS. La duración del protocolo experimental fue de aproximadamente 2 horas y todas las mediciones se llevaron a cabo entre las 16.00 y $18.00 \mathrm{~h}$, durante la fase descendente de la CAS. Los miembros de los grupos que recibieron alcohol permanecieron en las instalaciones hasta que su concentración de alcohol bajó a los límites legales para conducir.

La CAS fue 0,00 g/L para las mujeres y los hombres antes de tomar la bebida alcohólica, y la media fue 0,53 $\pm 0,12$ $\mathrm{g} / \mathrm{L}$ para las mujeres y $0,34 \pm 0,01 \mathrm{~g} / \mathrm{L}$ para los hombres después de tomar la bebida alcohólica. Es importante señalar que, si bien todos los sujetos consumieron la misma cantidad de alcohol, las diferencias estadísticas en CAS entre mujeres y hombres no permitieron una comparación directa entre sexos; sin embargo, fue posible estudiar los efectos del alcohol dentro de cada sexo (Experimento 1). Se llevó a cabo una selección contrabalanceada de mujeres y hombres con una CAS similar de 0,38 $\pm 0,01 \mathrm{~g} / \mathrm{L}$ para estudiar el factor género (Experimento 2). La Tabla 1 resu- 
Tabla 1. Características de la población de estudio (Experimento 1).

\begin{tabular}{|c|c|c|c|c|c|c|}
\hline & \multirow{2}{*}{\multicolumn{3}{|c|}{ Abstemios (ingesta ocasional) $(n=38)$}} & & & \\
\hline & & & & \multicolumn{3}{|c|}{ Binge Drinkers $(\mathrm{n}=130)$} \\
\hline & $\begin{array}{l}\text { Mujeres } \\
(\mathrm{n}=21)\end{array}$ & $\begin{array}{l}\text { Hombres } \\
(n=17)\end{array}$ & $\begin{array}{c}\text { Mujeres + } \\
\text { Hombres } \\
(n=38)\end{array}$ & $\begin{array}{l}\text { Mujeres } \\
(n=81)\end{array}$ & $\begin{array}{c}\text { Hombres } \\
(n=49)\end{array}$ & $\begin{array}{l}\text { Mujeres + Hombres } \\
\quad(n=130)\end{array}$ \\
\hline Edad al primer consumo de alcohol & $15,809 \pm 0,255$ & $16,058 \pm 0,326$ & $15,921 \pm 0,185$ & $14,654 \pm 0,125 \& \&$ & $14,714 \pm 0,157 \& \&$ & $14,676 \pm 0,097++$ \\
\hline Número medio de ocasiones por mes & $0,565 \pm 0,146$ & $0,482 \pm 0,155$ & $0,528 \pm 0,097$ & $2,666 \pm 0,084 \& \&$ & $2,612 \pm 0,119 \& \&$ & $2,646 \pm 0,068++$ \\
\hline Número medio de bebidas por ocasión & $2,952 \pm 0,381$ & $2,0 \pm 0,332$ & $2,562 \pm 0,246$ & $6,259 \pm 0,232 \& \&$ & $7,265 \pm 0,325 \& \&$ & $6,638 \pm 0,194++$ \\
\hline $\begin{array}{l}\text { Número medio de episodios de BD por } \\
\text { mes }\end{array}$ & NA & NA & NA & $2,666 \pm 0,084$ & $2,612 \pm 0,119$ & $2,646 \pm 0,068$ \\
\hline \multirow[t]{3}{*}{$\begin{array}{l}\text { Duración media del patrón BD (en } \\
\text { meses) hasta el inicio del experimento }\end{array}$} & NA & NA & NA & $11,333 \pm 0,262$ & $11,326 \pm 0,267$ & $11,330 \pm 0,084$ \\
\hline & \multicolumn{3}{|c|}{$\begin{array}{l}\text { Abstemios (abstemios puros + consumidores } \\
\text { ocasionales) }(n=105)\end{array}$} & \multicolumn{3}{|c|}{ Binge Drinkers $(n=130)$} \\
\hline & $\begin{array}{l}\text { Mujeres } \\
(\mathrm{n}=62)\end{array}$ & $\begin{array}{c}\text { Hombres } \\
(n=43)\end{array}$ & $\begin{array}{c}\text { Mujeres + } \\
\text { Hombres } \\
(\mathrm{n}=105)\end{array}$ & $\begin{array}{l}\text { Mujeres } \\
(\mathrm{n}=81)\end{array}$ & $\begin{array}{c}\text { Hombres } \\
(n=49)\end{array}$ & $\begin{array}{l}\text { Mujeres + Hombres } \\
\quad(n=130)\end{array}$ \\
\hline $\begin{array}{l}\text { Número medio de bebidas estimulantes: } \\
\text { cola, té o café/día }\end{array}$ & $1,048 \pm 0,160$ & $0,953 \pm 0,188$ & $1,009 \pm 0,121$ & $0,950 \pm 0,100$ & $0,714 \pm 0,148$ & $0,861 \pm 0,084$ \\
\hline Fumador: no/sí & $62 / 0$ & $43 / 0$ & $105 / 0$ & $61 / 20$ & $42 / 7$ & $83 / 27$ \\
\hline Evento estresante en el último año: no/sí & $47 / 15$ & $38 / 5$ & $85 / 20$ & $63 / 18$ & $44 / 5$ & $87 / 23$ \\
\hline Nervioso/a: no/sí & $45 / 17$ & $34 / 8$ & $79 / 25$ & $73 / 8$ & $42 / 5$ & $97 / 13$ \\
\hline Buen descanso: no/sí & $6 / 56$ & $6 / 37$ & $12 / 93$ & $9 / 72$ & $4 / 45$ & $13 / 97$ \\
\hline Actividad deportiva: no/sí & $53 / 9$ & $39 / 4$ & $92 / 13$ & $66 / 15$ & $36 / 13$ & $82 / 28$ \\
\hline Desayuno: no/sí & $9 / 53$ & $7 / 36$ & $16 / 89$ & $13 / 68$ & $5 / 44$ & $18 / 92$ \\
\hline
\end{tabular}

Los resultados están expresados como número o media \pm SEM para Abstemios y Binge Drinkers. NA: no aplicable.

$\& \& p<, 001 v s$ consumidores ocasionales en el mismo sexo. $++p<, 001$ vs consumidores ocasionales.

me las características de la población de estudio (representada en su totalidad en el Experimento 1).

\section{Análisis estadístico}

Los datos para mujeres y hombres se analizaron por separado, ya que la CAS fue estadísticamente diferente en los dos grupos (Experimento 1), y de forma conjunta cuando la CAS fue similar (Experimento 2). Los datos se sometieron a un análisis paramétrico tras confirmar que cumplían con los criterios de normalidad y homogeneidad de las varianzas. Se establecieron diferencias estadísticamente significativas en $p<, 05$ y la potencia estadística se calculó usando alfa $=0,05$. Se realizó un ANOVA de medidas repetidas para cortisol COR20' y COR50' (la medida COR0' no se incluyó en este ANOVA porque el primer registro de cortisol fue tomado antes de administrar el tratamiento de bebida de alcohol o de control). Se realizó un ANOVA de una vía para cada medida de respuesta al estrés (PAS, PAD, FC y EP) para mujeres y hombres, por separado (Experimento 1) o juntos (Experimento 2). Cada análisis contuvo los factores intersujeto 'Patrón de consumo' (abstemios y binge drinkers) y 'Tratamiento' (bebida de control y bebida de alcohol) como variables independientes. El factor 'Sexo' también se incluyó como un tercer factor intersujeto en el Experimento 2. Cuando cualquier interacción en- tre estos factores era estadísticamente significativa, se llevaron a cabo comparaciones por pares mediante la prueba $t$ de Student. Se exploraron todas las correlaciones de las medidas de respuesta al estrés registradas a los 20 minutos del tratamiento. Todos los análisis se realizaron utilizando el paquete de software SPSS Statistics, versión 26 para Windows (IBM, 2019).

\section{Resultados}

Experimento 1: Efectos de una dosis de riesgo de alcohol sobre el cortisol, la PA, la FC y el EP en mujeres (CAS: 0,53 \pm 0,12 $\mathrm{g} / \mathrm{L}$ ) y hombres (CAS: 0,34 $\pm 0,01 \mathrm{~g} / \mathrm{L}$ ) adolescentes con o sin antecedentes de $\mathrm{BD}$.

La Tabla 2 resume las estadísticas descriptivas y los resultados de los principales factores en las respuestas al estrés en el Experimento 1.

\section{Cortisol (CORo', COR20' y COR50')}

El factor Patrón de Consumo en COR0' no fue estadísticamente significativo, ni en mujeres $\left(F_{(1,79)}=0,122, p=\right.$ ,727) ni en hombres $\left(F_{(1,53)}=2,308, p=, 135\right)$.

COR20' y COR50': El ANOVA con medidas repetidas muestra que el Cortisol fue estadísticamente significativo en mujeres $\left(F_{(1,77)}=16,842, p=, 001\right)$ y hombres $\left(F_{(1,51)}=32,155\right.$, 
Desregulación del eje hipotalámico-pituitario-adrenal iniciada por un patrón binge drinking, pero no por el consumo agudo de alcohol, en mujeres y hombres adolescentes

Tabla 2. Resumen de estadísticas descriptivas y resultados para COR, PAS, PAD, FC y EP (Experimento 1).

\begin{tabular}{|c|c|c|c|c|c|c|}
\hline & \multicolumn{6}{|c|}{ MUJERES (CAS: $0,53 \pm 0,12 \mathrm{~g} / \mathrm{L}$ ) } \\
\hline & \multicolumn{3}{|c|}{ Patrón de consumo } & \multicolumn{3}{|c|}{ Tratamiento } \\
\hline & Abstemios & Binge Drinkers & Poder Estadístico $^{1}$ & Control & Alcohol & Poder Estadístico $^{1}$ \\
\hline $\operatorname{COR}(n=81)$ & $4,59 \pm 0,34$ & $4,77 \pm 0,37$ & ,982 & $4,18 \pm 0,29$ & $5,45 \pm 0,42+$ & ,982 \\
\hline PAS $(n=143)$ & $106,45 \pm 1,32$ & $104,49 \pm 1,09$ & ,219 & $104,58 \pm 1,14$ & $106,24 \pm 1,25$ & ,137 \\
\hline PAD $(n=143)$ & $68,96 \pm 0,97$ & $68,14 \pm 0,85$ &, 068 & $68,78 \pm 0,81$ & $68,16 \pm 0,94$ & ,088 \\
\hline FC $\quad(n=138)$ & $72,36 \pm 1,20$ & $77,05 \pm 1,11 \#$ & ,656 & $72,93 \pm 1,01$ & $77,55 \pm 1,33^{*}$ & ,659 \\
\hline \multirow[t]{4}{*}{ EP $\quad(n=137)$} & $24,67 \pm 0,93$ & $24,08 \pm 0,86$ & 109 & $23,26 \pm 0,83$ & $25,50 \pm 0,94$ & ,409 \\
\hline & \multicolumn{6}{|c|}{ HOMBRES (CAS: $0,34 \pm 0,01 \mathrm{~g} / \mathrm{L})$} \\
\hline & \multicolumn{3}{|c|}{ Patrón de consumo } & \multicolumn{3}{|c|}{ Tratamiento } \\
\hline & Abstemios & Binge Drinkers & Poder Estadístico $^{1}$ & Control & Alcohol & Poder Estadístico $^{1}$ \\
\hline $\operatorname{COR}(n=55)$ & $5,89 \pm 0,54$ & $7,88 \pm 0,84 \$$ & 1,000 & $7,37 \pm 0,57$ & $6,11 \pm 0,85$ & 1,000 \\
\hline PAS $(n=86)$ & $116,90 \pm 1,71$ & $122,39 \pm 1,60 \&$ & ,314 & $118,87 \pm 1,81$ & $121,00 \pm 1,51$ &, 069 \\
\hline PAD $(n=86)$ & $67,32 \pm 1,37$ & $71,06 \pm 1,37$ & ,424 & $68,00 \pm 1,45$ & $70,92 \pm 1,59$ &, 218 \\
\hline FC $\quad(n=89)$ & $74,04 \pm 1,82$ & $71,25 \pm 1,42$ &, 332 & $72,04 \pm 1,49$ & $73,15 \pm 1,77$ &, 080 \\
\hline EP $\quad(n=92)$ & $18,39 \pm 1,15$ & $20,00 \pm 1,15$ & ,122 & $18,20 \pm 1,05$ & $20,50 \pm 1,27$ & ,148 \\
\hline
\end{tabular}

Notas. Concentraciones de cortisol en saliva (COR). Presión arterial sistólica (PAS) y diastólica (PAD). Frecuencia cardiaca (FC). Estrés percibido (EP). No aplicable (NA) Los resultados se expresan como medias + SEM. ${ }^{1} \mathrm{El}$ poder estadístico se calcula utilizando alfa $=0,05$. Mujeres: $+p<, 05$ aumento de cortisol en Grupo de alcohol vs Grupo de control. \# $p<, 05$ aumento de FC en Binge Drinkers vs Abstemios. * $p<, 05$ aumento de FC en Grupo de alcohol vs Grupo de control. Hombres: \$ $p<, 05$ aumento de cortisol en Binge Drinkers vs Abstemios. \& $p<, 05$ aumento de PAS en Binge Drinkers vs Abstemios.

$p=, 001)$, mostrando una disminución significativa de COR50' con respecto a COR20' tanto en mujeres como en hombres (Figura 1A). Las interacciones Cortisol X Patrón de Consumo, Cortisol X Tratamiento, y Cortisol X Patrón de Consumo X Tratamiento no fueron estadísticamente significativas, ni en las mujeres $\left(F_{(1,77)}=0,790, p=, 377\right),\left(F_{(1,77)}=\right.$ $0,472, p=, 494),\left(F_{(1,77)}=0,014, p=, 907\right)$, respectivamente; ni en hombres $\left(F_{(1,51)}=1,313, p=, 257\right),\left(F_{(1,51)}=0,823, p=, 369\right)$, $\left(F_{(1,51)}=0,551, p=, 461\right)$, respectivamente.

El factor principal Patrón de Consumo en las mujeres no resultó ser estadísticamente significativo $\left(F_{(1,77)}=0,629, p=\right.$ ,430), mientras que el factor Tratamiento fue estadísticamente significativo $\left(F_{(1,77)}=6,222, p=, 015\right)$, con niveles más altos de cortisol detectados en los grupos de bebida de alcohol

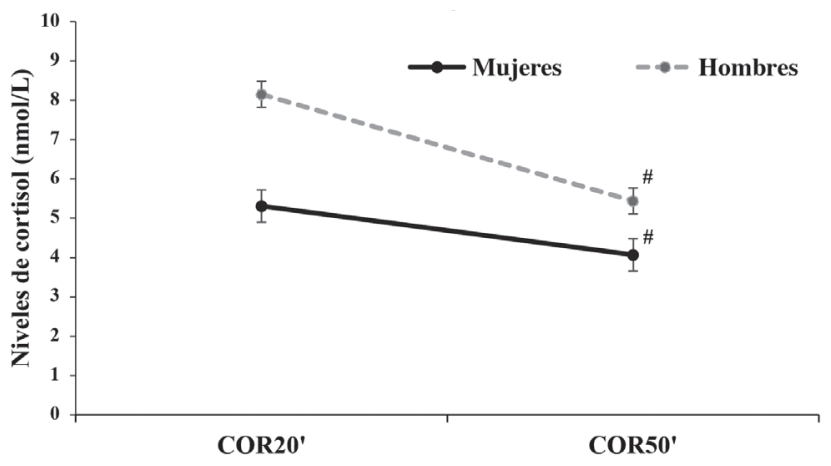

Figura $1 A$. Media de las concentraciones de cortisol salival a los 20 minutos (COR20') y 50 minutos (COR50') después del tratamiento en mujeres y hombres por separado (Experimento 1). \# $p<, 05$ vs COR20'.
(Tabla 2). La interacción Patrón de Consumo X Tratamiento no fue estadísticamente significativa $\left(F_{(1,77)}=0,739, p=, 393\right)$.

El factor principal Patrón de Consumo en los hombres fue estadísticamente significativo $\left(F_{(1,51)}=4,071, p=, 049\right)$, los binge drinkers mostraron niveles más altos de cortisol que los abstemios (Tabla 2); mientras que el Tratamiento no fue estadísticamente significativo $\left(F_{(1,51)}=2,151, p=, 149\right)$. La interacción Patrón de Consumo X Tratamiento no fue significativa $\left(F_{(1,51)}=0,932, p=, 339\right)$.

\section{Presión Arterial (PAS y PAD)}

PAS: En las mujeres, ni los factores principales -Patrón de Consumo $\left(F_{(1,139)}=1,379, p=, 242\right)$ y Tratamiento $\left(F_{(1,139)}\right.$ $=0,993, p=, 321)-$ ni su interacción $\left(F_{(1,139)}=1.491, p=\right.$ ,224) fueron estadísticamente significativos. En los hom-

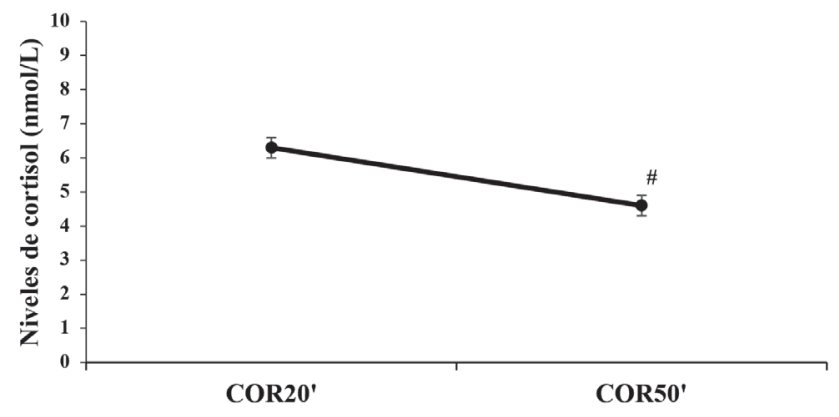

Figura 1B. Media de las concentraciones de cortisol salival a los 20 minutos (COR20') y 50 minutos (COR50') después del tratamiento en mujeres y hombres juntos (Experimento 2). \# $p<, 05$ vs COR20'. 
bres, el Patrón de Consumo fue estadísticamente significativo $\left(F_{(1,82)}=4,892, p=, 03\right)$, con los binge drinkers mostrando una PAS más alta que los abstemios (Tabla 2). Ni el factor Tratamiento ni la interacción Patrón de Consumo X Tratamiento fueron estadísticamente significativos $\left(F_{(1,82)}=\right.$ $0,660, p=, 419 ; F_{(1,82)}=0,256, p=, 615$; respectivamente).

PAD: En las mujeres, ni los factores principales -Patrón de Consumo $\left(F_{(1,139)}=0,165, p=, 685\right)$ y Tratamiento $\left(F_{(1,139)}=\right.$ $0,253, p=, 616))-$ ni su interacción $\left(F_{(1,139)}=0,729, p=, 395\right)$ fueron estadísticamente significativos. En los hombres, el Patrón de Consumo no fue estadísticamente significativo $\left(F_{(1,82)}\right.$ $=3,143, p=, 08)$, ni tampoco lo fueron el factor Tratamiento ni la interacción Patrón de Consumo X Tratamiento $\left(F_{(1,82)}=\right.$ $2,062, p=, 155 ; F_{(1,82)}=0,323, p=, 572$; respectivamente).

\section{Frecuencia cardíaca (FC)}

En las mujeres, el factor Patrón de Consumo fue estadísticamente significativo $\left(F_{(1,134)}=5,625, p=, 019\right)$, con las binge drinkers mostrando una FC más alta que las abstemias (Tabla 2). El factor Tratamiento también fue estadísticamente significativo $\left(F_{(1,134)}=5,588, p=, 02\right)$ entre las mujeres, las que bebieron alcohol presentaron una FC más alta (Tabla 2). La interacción Patrón de Consumo X Tratamiento no fue estadísticamente significativa en las mujeres $\left(F_{(1,134)}=0,016, p=, 898\right)$. En los hombres, ni el Patrón de Consumo $\left(F_{(1,85)}=2,319, p=, 132\right)$ ni el Tratamiento $\left(F_{(1,85)}=\right.$ ,605, $p=, 439)$ fueron estadísticamente significativos, mientras que la interacción Patrón de Consumo X Tratamiento sí lo fue $\left(F_{(1,85)}=4,877, p=, 03\right)$, mostrando los abstemios que bebieron alcohol una FC más alta que todos los demás grupos (A-Co, BD-Co y BD-A) (Figura 2).

\section{Estrés Percibido (EP)}

Ni los principales factores -Patrón de Consumo y Tratamiento- ni su interacción fueron estadísticamente significativos en las mujeres (Patrón de Consumo: $F_{(1,133)}=0,508$, $p=, 477$; Tratamiento: $F_{(1,133)}=3,057, p=, 083$; Interacción: $\left.F_{(1,133)}=0,140, p=, 709\right)$ ni en los hombres (Patrón de Con-

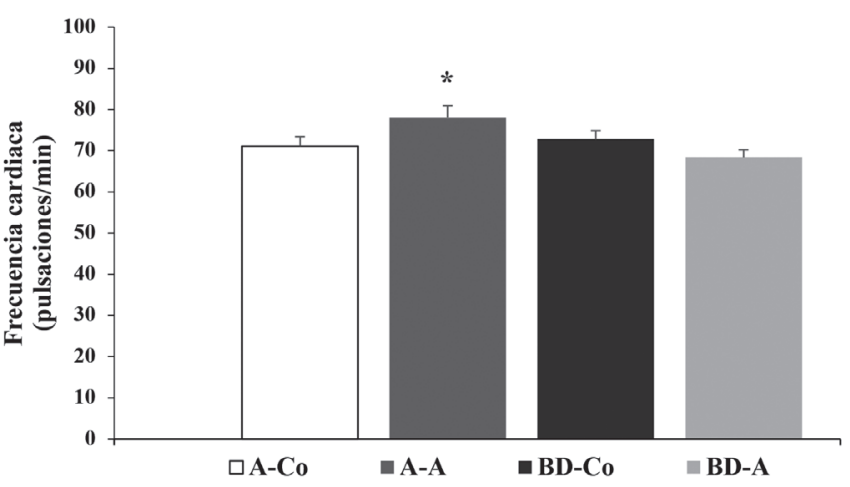

Figura 2. Frecuencia cardíaca (FC) en hombres en el Experimento 1. Los valores están expresados como medias (+ SEM). A-Co: Abstemios-Control; A-A: Abstemios-Alcohol; BD-Co: binge drinkers-control; BD-A: binge drinkers-alcohol. * $p<, 05$ vs A-Co, BD-Co y BD-A.

sumo: $F_{(1,88)}=0,633, p=, 428$; Tratamiento: $F_{(1,88)}=1,687$, $p=, 197$; Interacción: $\left.F_{(1,88)}=0,030, p=, 862\right)$.

\section{Correlaciones entre medidas}

Se detectaron correlaciones positivas en mujeres $20 \mathrm{~min}$ después del tratamiento, lo que demuestra un aumento mutuo de las siguientes variables: PAS y PAD $(r=0,617, p=$ ,001), y PAD y FC ( $r=0,222, p=, 046)$. También se encontraron correlaciones positivas en hombres entre COR20' y PAS ( $\mathrm{r}=0,344, p=, 013)$, PAS y PAD $(\mathrm{r}=0,417, p=, 002)$, $\mathrm{y}$ PAD y FC $(r=0,434, p=, 001)$.

Experimento 2: Efectos de una dosis de riesgo de alcohol sobre el cortisol, la PA, la FC y el EP en mujeres y hombres (CAS: 0,38 $\pm 0,01 \mathrm{~g} / \mathrm{L}$ ) adolescentes con o sin antecedentes de BD.

La Tabla 3 resume las estadísticas descriptivas y los resultados de los principales factores en las respuestas al estrés en el Experimento 2.

\section{Cortisol (CORo', COR2o' y COR50')}

En COR0', ni el factor Patrón de Consumo $\left(F_{(1,92)}=\right.$ $1,6470, p=, 203)$ ni el factor Sexo $\left(F_{(1,92)}=0,057, p=, 811\right)$

Tabla 3. Resumen de estadísticas descriptivas y resultados para COR, PAS, PAD, FC y EP (Experimento 2).

\begin{tabular}{|c|c|c|c|c|c|c|c|c|c|}
\hline & \multicolumn{9}{|c|}{ MUJERES Y HOMBRES (CAS: $0,38 \pm 0,01 \mathrm{~g} / \mathrm{L})$} \\
\hline & \multicolumn{3}{|c|}{ Patrón de consumo } & \multicolumn{3}{|c|}{ Tratamiento } & \multicolumn{3}{|c|}{ Sexo } \\
\hline & $\begin{array}{l}\text { Abstemios } \\
(\mathrm{n}=48)\end{array}$ & $\begin{array}{l}\text { Binge Drinkers } \\
\quad(n=48)\end{array}$ & $\begin{array}{c}\text { Poder } \\
\text { Estadístico }^{1}\end{array}$ & $\begin{array}{l}\text { Control } \\
(n=48)\end{array}$ & $\begin{array}{l}\text { Alcohol } \\
(n=48)\end{array}$ & $\begin{array}{c}\text { Poder } \\
\text { Estadístico }^{1}\end{array}$ & $\begin{array}{l}\text { Mujeres } \\
(n=48)\end{array}$ & $\begin{array}{l}\text { Hombres } \\
(n=48)\end{array}$ & $\begin{array}{c}\text { Poder } \\
\text { Estadístico }^{1}\end{array}$ \\
\hline COR & $4,94 \pm 0,37$ & $5,37 \pm 0,55$ & ,995 & $5,67 \pm 0,45$ & $5,23 \pm 0,50$ & ,995 & $4,77 \pm 0,32$ & $6,14 \pm 0,58 \#$ & ,995 \\
\hline PAS & $113,75 \pm 1,54$ & $113,60 \pm 1,86$ &, 051 & $114,29 \pm 1,80$ & $113,06 \pm 1,61$ & ,098 & $106,52 \pm 1,27$ & $120,83 \pm 1,44^{*}$ & 1,000 \\
\hline PAD & $68,70 \pm 0,92$ & $68,83 \pm 1,25$ &, 051 & $69,02 \pm 0,90$ & $68,52 \pm 1,22$ &, 062 & $68,08 \pm 0,93$ & $69,45 \pm 1,23$ & ,144 \\
\hline FC & $72,12 \pm 1,55$ & $74,93 \pm 1,46$ & ,278 & $70,33 \pm 1,41$ & $76,72 \pm 1,56 \$$ & ,875 & $75,16 \pm 1,38$ & $71,89 \pm 1,69$ & ,357 \\
\hline EP & $21,93 \pm 1,14$ & $23,12 \pm 1,28$ &, 083 & $21,77 \pm 1,22$ & $23,29 \pm 1,19$ & ,100 & $24,39 \pm 1,07 \&$ & $20,66 \pm 1,29$ &, 636 \\
\hline
\end{tabular}

Notas. Concentraciones de cortisol en saliva (COR). Presión arterial sistólica (PAS) y diastólica (PAD). Frecuencia cardiaca (FC). Estrés percibido (EP). No aplicable (NA). Los resultados se expresan como medias \pm SEM. ${ }^{1}$ El poder estadístico se calcula utilizando alfa $=0,05 . \# p<, 05$ aumento de COR vs mujeres. ${ }^{\star} p<, 05$ aumento de PAS vs mujeres. $\$ p<, 05$ aumento de FC en Grupo de alcohol vs Grupo de control. \& $p<, 05$ aumento de EP vs hombres. 
fueron estadísticamente significativos. Asimismo, la interacción Patrón de Consumo X Sexo no fue estadísticamente significativa $\left(F_{(1,92)}=0,004, p=, 948\right)$.

COR20' y COR50': El ANOVA de medidas repetidas mostró que el Cortisol fue estadísticamente significativo $(F(1,88)=20,764, p=, 001)$, mostrando una disminución significativa de COR50' con respecto a COR20' (Figura 1B). Ningunas de las interacciones posibles entre Cortisol, Patrón de Consumo, Tratamiento y Sexo fue estadísticamente significativa ( $p$ s $>, 05$ en todos los casos).

Los factores principales Patrón de Consumo y Tratamiento no resultaron ser estadísticamente significativos $\left(F_{(1,88)}=2,584, p=, 112 ;\right.$ y $F_{(1,88)}=0,460, p=, 500$; respectivamente); mientras que Sexo fue significativo $\left(F_{(1,88)}=4,538\right.$, $p=, 036)$, con niveles más altos de cortisol detectados en los grupos de hombres (Tabla 3). La interacción Patrón de Consumo X Tratamiento también fue estadísticamente significativa $\left(F_{(1,88)}=4,492, p=, 037\right)$, mostrando los binge drinkers que recibieron bebida de control niveles más altos de cortisol que el resto de los sujetos (Figura 3). Las interacciones restantes no fueron estadísticamente significativas (Patrón de Consumo X Sexo: $F_{(1,88)}=0,116, p=, 735$; Tratamiento X Sexo: $F_{(1,88)}=3,578, p=, 062$; y Patrón de Consumo X Tratamiento X Sexo: $\left.F_{(1,88)}=1,370, p=, 245\right)$.

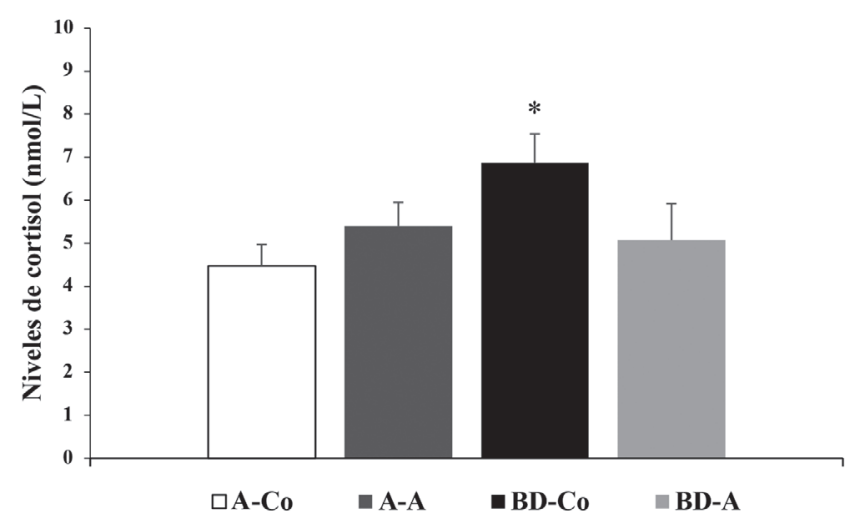

Figura 3. Concentraciones de cortisol salivales (mujeres y hombres juntos) en el Experimento 2. Los valores están expresados como medias (+ SEM). A-Co: Abstemios-Control; A-A: Abstemios-Alcohol; BD-Co: binge drinkerscontrol; BD-A: binge drinkers-alcohol. ${ }^{\star} p<, 05$ vs A-Co.

\section{Presión Arterial (PAS y PAD)}

PAS: No se observaron resultados significativos para los factores principales Patrón de Consumo $\left(F_{(1,88)}=0,006, p=\right.$ ,939) o Tratamiento $\left(F_{(1,88)}=0,418, p=, 520\right)$, pero el factor Sexo fue estadísticamente significativo $\left(F_{(1,88)}=56,639\right.$, $p=, 001)$, mostrando los hombres una PAS más alta que las mujeres (Tabla 3). La interacción Patrón de Consumo $\mathrm{X}$ Sexo fue estadísticamente significativa $\left(F_{(1,88)}=3,675\right.$, $p=, 05)$, obteniendo los hombres binge drinkers una PAS más alta que sus homólogas mujeres (Figura 4). Las interacciones Patrón de Consumo X Tratamiento $\left(F_{(1,88)}=1,273\right.$, $p=, 262)$, Tratamiento X Sexo $\left(F_{(1,88)}=0,640, p=, 426\right) \mathrm{y}$

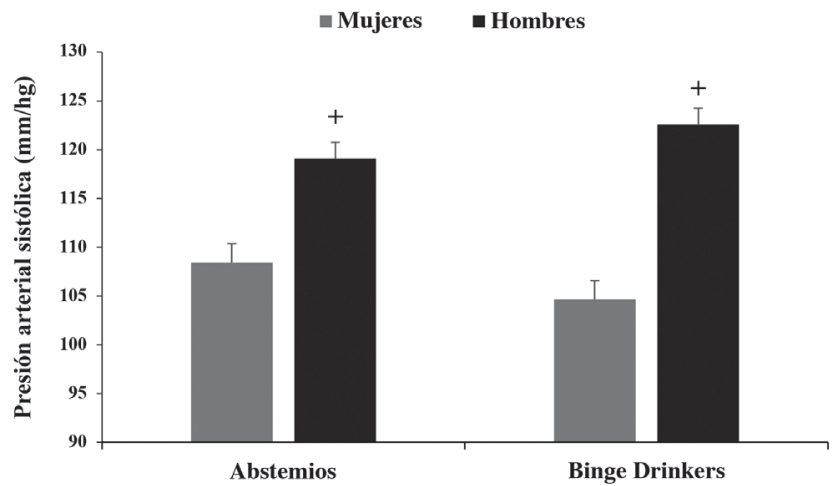

Figura 4. Presión arterial sistólica (PAS) en mujeres y hombres en el Experimento 2. Los valores están expresados como medias (+ SEM) $++p<$ ,05 vs mujeres.

Patrón de Consumo X Tratamiento X Sexo $\left(F_{(1,88)}=2,123\right.$, $p=, 149)$ no fueron estadísticamente significativas.

PAD: No se obtuvieron resultados significativos para los factores principales Patrón de Consumo $\left(F_{(1,88)}=0,007, p=\right.$ ,935), Tratamiento $\left(F_{(1,88)}=0,106, p=, 746\right)$ y Sexo $\left(F_{(1,88)}=\right.$ $0,802, p=, 373)$. Las interacciones Patrón de Consumo X Tratamiento $\left(F_{(1,88)}=3,816, p=, 054\right)$, Patrón de Consumo X Sexo $\left(F_{(1,88)}=3,305, p=, 072\right)$, Tratamiento X Sexo $\left(F_{(1,88)}=0,851\right.$, $p=, 359)$ y Patrón de Consumo X Tratamiento X Sexo $\left(F_{(1,88)}\right.$ $=0,027, p=, 871)$ no fueron estadísticamente significativas.

\section{Frecuencia cardíaca (FC)}

La FC no fue estadísticamente significativa para los factores principales Patrón de Consumo $\left(F_{(1,88)}=1,915, p=\right.$ ,170) y Sexo $\left(F_{(1,88)}=2,590, p=, 111\right)$, pero lo fue para el factor Tratamiento $\left(F_{(1,88)}=9,902, p=, 002\right)$, con mayor FC observada entre los sujetos que recibieron alcohol durante el experimento (Tabla 3). La interacción Patrón de Consumo X Tratamiento no fue estadísticamente significativa $\left(F_{(1,88)}=3.442, p=, 067\right)$, pero la interacción Patrón de Consumo X Sexo sí lo fue $\left(F_{(1,88)}=4,245, p=, 042\right)$, observándose una mayor FC en las mujeres binge drinkers vs los demás grupos (Figura 5). El resto de interacciones no fueron estadísticamente significativas (Tratamiento X Sexo: $F_{(1,88)}=$ 0,318, $p=, 574$; Patrón de Consumo X Tratamiento X Sexo: $\left.F_{(1,88)}=1,342, p=, 250\right)$.

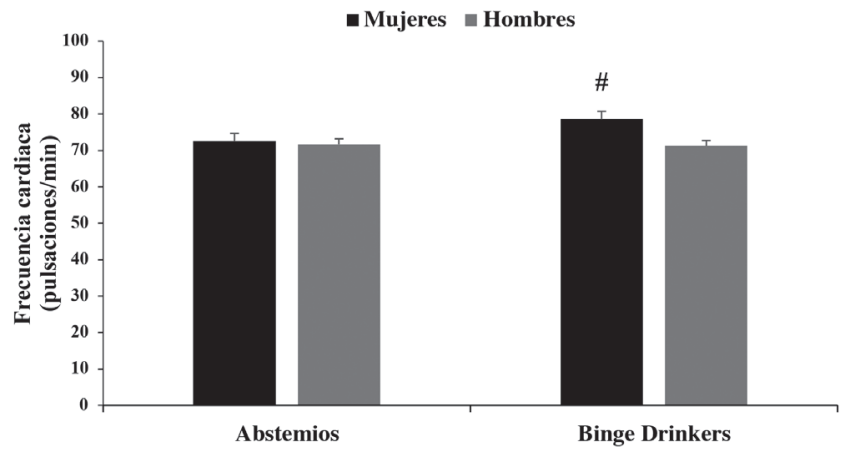

Figura 5. Frecuencia cardíaca (FC) en mujeres y hombres en el Experimento 2. Los valores están expresados como medias (+ SEM). \# $p<, 05$ vs todos los grupos. 


\section{Estrés Percibido (EP)}

Los resultados obtenidos para EP no fueron estadísticamente significativos para los factores Patrón de Consumo $\left(F_{(1,88)}=0,488, p=, 487\right)$ o Tratamiento $\left(F_{(1,88)}=0,800, p=\right.$ $, 373)$, pero lo fue para el factor Sexo $\left(F_{(1,88)}=4,811, p=\right.$ ,031), con las mujeres obteniendo puntuaciones más altas en EP que los hombres (Tabla 3). Ninguna de las interacciones fue estadísticamente significativa (Patrón de Consumo X Tratamiento: $F_{(1,88)}=0,523, p=, 472$; Patrón de Consumo X Sexo: $F_{(1,88)}=0,025, p=, 874$; Tratamiento X Sexo: $F_{(1,88)}=0,109, p=, 742 ;$ Patrón de Consumo X Tratamiento X Sexo: $\left.F_{(1,88)}=1,243, p=, 268\right)$.

\section{Correlaciones entre medidas}

Se detectaron correlaciones positivas $20 \mathrm{~min}$ después del tratamiento entre las siguientes variables: COR20' y PAS $(r=0,314, p=, 002)$, PAS y PAD $(r=0,493, p=, 001), y$ PAD y FC $(\mathrm{r}=0,235, p=, 021)$.

\section{Discusión}

Este estudio fue diseñado para clarificar los efectos que tiene una historia de BD y/o los efectos del consumo agudo de alcohol sobre la funcionalidad del eje HPA (cortisol) y otras variables de la respuesta al estrés (FC, PA y EP) en mujeres y hombres adolescentes. Nuestra hipótesis era que habría un aumento de los niveles de cortisol, FC, PA y EP en los participantes sin antecedentes de BD, debido a la respuesta normal al estrés del consumo agudo de alcohol (Hagan et al., 2019; King et al., 2006; Lu y Richardson, 2014; Ulrich-Lai y Herman, 2009). Nuestros resultados parcialmente apoyan esta hipótesis: el cortisol y la FC fueron más altos en las mujeres que bebieron alcohol durante el Experimento 1 y la FC fue más alta entre los participantes de ambos sexos que bebieron alcohol durante el Experimento 2. Los resultados de nuestro estudio son coherentes con investigaciones anteriores que muestran que el alcohol influye directamente en el eje HPA al elevar los niveles de la hormona cortisol (King et al., 2006; Lu y Richardson, 2014; Richardson, Lee, O’Dell, Koob y Rivier, 2008). Aunque no se observó en nuestro estudio una correlación significativa entre el cortisol y la FC, el aumento de la actividad cardiovascular en las mujeres podría deberse al aumento de los niveles de la hormona HPA (cortisol), ya que otros grupos han informado de tal hallazgo (Bau et al., 2011; Sher et al., 2007).

Nuestros resultados tras la ingesta aguda de alcohol se relacionan con un sistema normal de respuesta al estrés, en coherencia con otros estudios (King et al., 2006; Lu y Richardson, 2014; Richardson et al., 2008). Además, nuestros resultados muestran que una historia de $\mathrm{BD}$ (no los efectos de la ingesta aguda de alcohol) también pueden tener un efecto significativo, ya que algunas variables del sistema de respuesta al estrés aparentemente ya mostraban desregula- ción debido a episodios anteriores de BD. De esta manera, nuestros resultados en el Experimento 1 revelaron niveles más altos de FC en mujeres $\mathrm{BD}$, y niveles basales más altos de cortisol y PAS en hombres BD. En el Experimento 2, los niveles basales de cortisol eran más altos en los participantes $\mathrm{BD}$ que recibieron bebida de control en comparación con los otros grupos. Esto sugiere que los adolescentes con una historia de BD (sin considerar cualquier ingesta aguda de alcohol) tienen niveles más altos de cortisol debido a la sobrerregulación del eje HPA (Wemm et al., 2013). Por tanto, hemos cumplido el otro objetivo principal de nuestro estudio al demostrar que el BD desencadena cambios en el eje HPA y en la respuesta al estrés en adolescentes sanos que iniciaron un patrón de consumo BD en la adolescencia. En contraste con nuestros hallazgos, otros estudios han informado de valores más bajos de cortisol, FC, PA y $\mathrm{EP}$ en sujetos con antecedentes de BD que participan en el consumo agudo de alcohol, argumentando que el sistema de respuesta está regulado por una historia sostenida de BD (e.g., Hagan et al., 2019; Orio et al., 2018). No se observó esto en nuestro estudio, tal vez porque nuestros participantes no habían alcanzado ese punto de regulación: es decir, un historial de un año de BD no fue lo suficientemente largo para lograr la regulación antes mencionada (niveles más bajos en las medidas de respuesta al estrés).

Las interacciones que observamos son coherentes con los demás resultados de nuestro estudio, apuntando a un aumento de la FC en los hombres abstemios que bebieron alcohol, como han informado estudios anteriores (Bau et al., 2011; Sher et al., 2007; Vinader-Caerols, et al., 2012). Este resultado, observado en el Experimento 1, puede interpretarse como un fenómeno de tolerancia entre los binge drinkers masculinos, ya que su FC no aumentó cuando bebieron alcohol. En el Experimento 2, la interacción Patrón de Consumo X Tratamiento reveló niveles más altos de cortisol en el grupo BD que recibió la bebida de control, en comparación con los otros grupos. Esto confirma la sobrerregulación del estado basal del eje HPA informado en un estudio anterior (Wemm et al., 2013) y aporta evidencia de que el patrón BD desencadena alteraciones en el eje HPA, independientemente del sexo.

El objetivo principal del Experimento 2 fue estudiar directamente el factor sexo. Teniendo en cuenta que las variaciones de cortisol a lo largo del tiempo, observadas en nuestro estudio, van en la misma dirección en ambos sexos (niveles más bajos de COR50' que de COR20' en mujeres y hombres por separado, así como en ambos sexos juntos), la principal diferencia de sexo consistió en niveles más altos de cortisol en los hombres, quizás porque los aumentos del cortisol en saliva son hasta dos veces más altos en los hombres que en las mujeres. La literatura apoya esta diferencia de sexo: se ha demostrado que la respuesta media típica en los hombres varía entre 200 y $400 \%$ con respecto a la línea base, mientras que suelen verse cambios entre 50 y $150 \%$ 
en las mujeres (Kudielka, Hellhammer y Wüst, 2009). Otra diferencia de sexo se observó en el caso de la PA, con la PAS siendo más elevada en los hombres que en las mujeres. Según investigaciones anteriores, la PA de mujeres jóvenes suele ser más baja que la de hombres jóvenes, incluso entre personas normotensas sanas (Joyner, Wallin y Charkoudian, 2016). Además, la PAS fue más alta entre nuestros participantes hombres BD en comparación con las mujeres BD y los grupos de abstemios. Por último, nuestras mujeres participantes mostraron niveles más altos de EP que los hombres y el grupo de mujeres BD mostró una FC más alta que sus homólogos masculinos, según otros estudios (Anbumalar, Dorathy, Jaswanti, Priya y Reniangelin, 2017; Hogan, Carlson y Dua, 2002).

Varios estudios han informado que el cortisol facilita un aumento de la actividad cardiovascular, elevando la FC y la PA (Hagan et al., 2019; Ulrich-Lai y Herman, 2009). Los hallazgos de nuestro estudio respaldan parcialmente esta cuestión a través de correlaciones positivas entre los niveles de cortisol y la actividad cardiovascular (COR20' y PAS).

Entre las limitaciones de este estudio, debemos mencionar que medimos los niveles de cortisol después del tratamiento, pero no los medimos temprano por la mañana, cuando los niveles son los más elevados debido al ritmo circadiano de las variaciones de cortisol (Weitzman et al., 1971; Yamanaka, Motoshima y Uchida, 2019). Sin embargo, las muestras de cortisol en saliva estaban dentro del rango normal de valores basales $(2,7586-8,2758 \mathrm{nmol} / \mathrm{L})$ en el momento de su toma (16:00h-18:00h) (Aardal y Holm, 1995). Además, habría sido deseable una muestra más grande en el segundo experimento para respaldar los resultados obtenidos en mujeres y hombres con una CAS similar. Por último, es necesario realizar estudios longitudinales en el futuro para estudiar los efectos a largo plazo de la desregulación del eje HPA.

Considerando todos los resultados, y a pesar de las limitaciones antes mencionadas, nuestro estudio infiere que:

a) Un eje HPA normal en los adolescentes reacciona al consumo de alcohol aumentando los niveles de cortisol en las mujeres, así como la FC en ambos sexos, como reacción normal del sistema de respuesta al estrés.

b) Se observan valores más altos de cortisol (independientemente de sexo), FC en las mujeres y PAS en los hombres en sujetos con antecedentes de $\mathrm{BD}$ sostenida al menos durante un año, incluso si son adolescentes sanos, debido a una emergente desregulación en el eje HPA. Sin embargo, estos cambios son insuficientes para diagnosticar una enfermedad.

c) Las principales diferencias de sexo se encontraron en cortisol, PAS y EP, así los hombres mostraron niveles más altos de cortisol y PAS, y un EP más bajo, que las mujeres.

d) Nuestros resultados contribuyen a un mejor conocimiento de los cambios relacionados con el consumo de alcohol que ocurren en esta etapa de la vida (adolescencia tardía) y pueden ser útiles para el diseño de estrategias para prevenir los cambios en el eje HPA desencadenados por el BD, considerando el factor sexo y, por tanto, implementando programas de prevención más efectivos dirigidos a este grupo de riesgo.

\section{Reconocimientos}

Este trabajo contó con el apoyo de la Universitat de València (Proyecto UV-INV_AE18-779336) y la Generalitat Valenciana (Proyecto PROMETEO-II/2015/020), España.

Los autores desean agradecer al Sr. Brian Normanly su ayuda editorial.

\section{Conflicto de intereses}

Los autores declaran la inexistencia de conflicto de intereses.

\section{Referencias}

Allen, C. D., Lee, S., Koob, G. F. y Rivier, C. (2011). Immediate and prolonged effects of alcohol exposure on the activity of the hypothalamic-pituitary-adrenal axis in adult and adolescent rats. Brain, Behavior, and Immunity, 25 (Supl. 1), 50-60. doi:10.1016/j.bbi.2011.01.016.

Aardal, E. y Holm, A. C. (1995). Cortisol in saliva-reference ranges and relation to cortisol in serum. European Journal of Clinical Chemistry and Clinical Biochemistry, 33, 927932. doi:10.1515/cclm.1995.33.12.927.

Anbumalar, C., Dorathy, A. P., Jaswanti, V. P., Priya, D. y Reniangelin, D. (2017). Gender differences in perceived stress levels and coping strategies among college students. The International Journal of Indian Psychology, 4, 22-33. doi:10.25215/0404.103.

Bau, P. F., Moraes, R. S., Bau, C. H., Ferlin, E. L., Rosito, G. A. y Fuchs, F. D. (2011). Acute ingestion of alcohol and cardiac autonomic modulation in healthy volunteers. Alcohol, 45, 123-129. doi:10.1016/j.alcohol.

Becker, H. C. (2017). Influence of stress associated with chronic alcohol exposure on drinking. Neuropharmacolgy, 122, 115-126. doi:10.1016/j.neuropharm.2017.04.028.

Bidwal, M. K., Ip, E. J., Shah, B. M. y Serino, M. J. (2015). Stress, drugs, and alcohol use among health care professional students: A focus on prescription stimulants. Journal of Pharmacy Practice, 28, 535-542. doi:10.1177/0897190014544824.

Blaine, S. K. y Sinha, R. (2017). Alcohol, stress, and glucocorticoids: From risk to dependence and relapse in alcohol use disorders. Neuropharmacology, 122, 136-147. doi:10.1016/j.neuropharm.2017.01.037. 
Cohen, S., Kamarck, T. y Mermelstein, R. (1983). A global measure of perceived stress. Journal of Health and Social Behavior, 24, 385-396. doi:10.2307/2136404.

González-Cabrera, J., Fernández-Prada, M., Iribar-Ibabe, C. y Peinado, J. M. (2014). Acute and chronic stress increase salivary cortisol: A study in the real-life setting of a national examination undertaken by medical graduates. Stress, 17, 149-156. doi:10.3109/10253890.2013.876405.

Hagan, M. J., Modecki, K., Tan, L. M., Luecken, L., Wolchik, S. y Sandler, I. (2019). Binge drinking in adolescence predicts an atypical cortisol stress response in young adulthood. Psychoneuroendocrinology, 100, 137-144. doi:10.1016/j.psyneuen.2018.10.002.

Hayibor, L. A., Zhang, J. y Duncan, A. (2019). Association of binge drinking in adolescence and early adulthood with high blood pressure: Findings from the national longitudinal study of adolescent to adult health (19942008). Journal of Epidemiology and Community Health, 73, 652-659. doi:10.1136/jech-2018-211594.

Hellhammer, J. y Schubert, M. (2012). The physiological response to trier social stress test relates to subjective measures of stress during but not before or after the test. Psychoneuroendocrinology, 37, 119-124. doi:10.1016/j. psyneuen.20.

Hogan, J. M., Carlson, J. G. y Dua, J. (2002). Stressors and stress reactions among university personnel. International Journal of Stress Management, 9, 289-310.

IBM Corp Released. (2019). IBM SPSS Statistics for Windows, Version 26.0. Armonk, New York: IBM Corp.

Joyner, M. J., Wallin, B. G. y Charkoudian, N. (2016). Sex differences and blood pressure regulation in humans. Experimental Physiology, 101, 349-355. doi:10.1113/ EP085146.

Keyes, K. M., Hatzenbuehler, M. L., Grant, B. F. y Hasin, D. S. (2012). Stress and alcohol: Epidemiologic evidence. Alcohol Research: Current Reviews, 34, 391-400.

King, A. C., Houle, T., de Wit, H., Holdstock, L. y Schuster, A. (2002). Biphasic alcohol response differs in heavy versus light drinkers. Alcoholism, Clinical and Experimental Research, 26, 827-835. doi:10.1111/j.1530-0277.2002. tb02611.x.

King, A., Munisamy, G., de Wit, H. y Lin, S. (2006). Attenuated cortisol response to alcohol in heavy social drinkers. International Journal of Psychophysiology, 59, 203209. doi:10.1016/j.ijpsycho.2005.10.008.

Kudielka, B. M., Hellhammer, D. H. y Wüst, S. (2009). Why do we respond so differently? Reviewing determinants of human salivary cortisol responses to challenge. Psychoneuroendocrinology, 34, 2-18. doi:10.1016/j.psyneuen.2008.10.004.

Lee, S. K. (2018). Sex as an important biological variable in biomedical research. BMB Reports, 51, 167-173. doi:10.5483/bmbrep.2018.51.4.034.
López-Caneda, E., Mota, N., Crego, A., Velasquez, T., Corral, M., Rodríguez Holguín, S. y Cadaveira, F. (2014). Neurocognitive anomalies associated with the binge drinking pattern of alcohol consumption in adolescents and young people: A review. Adicciones, 26, 334-359.

Lu, Y. L. y Richardson, H. N. (2014). Alcohol, stress hormones, and the prefrontal cortex: A proposed pathway to the dark side of addiction. Neuroscience, 277, 139-151. doi:10.1016/j.neuroscience.2014.06.053.

Magrys, S. A., Olmstead, M. C., Wynne-Edwards, K. E. y Balodis, I. M. (2013). Neuroendocrinological responses to alcohol intoxication in healthy males: Relationship with impulsivity, drinking behavior, and subjective effects. Psychophysiology, 50, 204-209. doi:10.1111/psyp.12007.

National Institute on Alcohol Abuse and Alcoholism (NIAAA) (2004). The National Institute on Alcohol Abuse and Alcoholism council aproves definition of binge drinking. NIAAA Newsletter, 3, 3.

Observatorio Español de las Drogas y las Adicciones (OEDA) (2020). Encuesta sobre Uso de Drogas en Enseñanzas Secundarias en España (ESTUDES) 1994-2020. Madrid: Ministerio de Sanidad, Consumo y Bienestar Social.

Orio, L., Antón, M., Rodríguez-Rojo, I. C., Correas, Á., García-Bueno, B., Corral, M.,... Cadaveira, F. (2018). Young alcohol binge drinkers have elevated blood endotoxin, peripheral inflammation and low cortisol levels: Neuropsychological correlations in women. Addiction Biology, 23, 1130-1144. doi:10.1111/adb.12543.

Parada, M., Corral, M., Caamaño-Isorna, F., Mota, N., Crego, A., Rodríguez Holguín, S. y Cadaveira, F. (2011). Definición del concepto de consumo intensivo de alcohol adolescente (binge drinking). Adicciones, 23, 53-63. doi:10.20882/adicciones.167.

Richardson, H. N., Lee, S. Y., O’Dell, L. E., Koob, G. F. y Rivier, C. L. (2008). Alcohol self-administration acutely stimulates the hypothalamic-pituitary-adrenal axis, but alcohol dependence leads to a dampened neuroendocrine state. The European Journal of Neuroscience, 28, 16411653. doi:10.1111/j.1460-9568.2008.06455.x.

Saunders, J. B., Aasland, O. G., Babor, T. F., de La Fuente, J. R. y Grant, M. (1993). Development of the alcohol use disorders identification test (AUDIT): WHO collaborative project on early detection of persons with harmful alcohol consumption-II. Addiction, 88, 791-804. doi:10.1111/j.1360-0443.1993.tb02093.x.

Sher, K. J., Bartholow, B. D., Peuser, K., Erickson, D. J. y Wood, M. D. (2007). Stress-response-dampening effects of alcohol: Attention as a mediator and moderator. Journal of Abnormal Psychology, 116, 362-377. doi:10.1037/0021-843X.116.2.

Tavolacci, M. P., Ladner, J., Grigioni, S., Richard, L., Villet, H. y Dechelotte, P. (2013). Prevalence and association of perceived stress, substance use and behavioral addictions: A cross-sectional study among university students 
in France. BMC Public Health, 13, 724. doi:10.1186/14712458-13-724.

Ulrich-Lai, Y. M. y Herman, J. P. (2009). Neural regulation of endocrine and autonomic stress responses. Neuroscience, 10, 397-409. doi:10.1038/nrn2647.

Vinader-Caerols, C. y Monleón, S. (2019). Binge drinking and memory in adolescents and young adults. En S. Palermo y M. Bartoli (Eds.), Inhibitory Control Training-A Multidisciplinary Approach (pp.1-19). IntechOpen. doi:10.5772/intechopen.88485.

Vinader-Caerols, C., Monleón, S., Carrasco, C. y Parra, A. (2012). Effects of alcohol, coffee, and tobacco, alone or in combination, on physiological parameters and anxiety in a young population. Journal of Caffeine Research, 2, 70-76. doi:10.1089/jcr.2012.0018.

Vinader-Caerols, C., Talk, A., Montañés, A., Duque, A. y Monleón, S. (2017a). Differential effects of alcohol on memory performance in adolescent men and women with a binge drinking history. Alcohol and Alcoholism, 52, 610-616. doi:10.1093/alcalc/agx040.

Vinader-Caerols, C., Duque, A., Montañés, A. y Monleón, S. (2017b). Blood alcohol concentration-related lower performance in immediate visual memory and working memory in adolescent binge drinkers. Frontiers in Psychology, 8, 1720. doi:10.3389/fpsyg.2017.01720.

Waltman, C., Blevins, L. S., Boyd, G. y Wand, G. S. (1993). The effects of mild ethanol intoxication on the hypothalamic-pituitary-adrenal axis in nonalcoholic men. The Journal of Clinical Endocrinology and Metabolism, 77, 518522. doi:10.1210/jcem.77.2.8393888.

Weera, M. M. y Gilpin, N. W. (2019). Biobehavioral interactions between stress and alcohol. Alcohol Research: Current Reviews, 40, 04. doi:10.35946/arcr.v40.1.04.

Weitzman, E. D., Fukushima, D., Nogeire, C., Roffwarg, H., Gallagher, T. F. y Hellman, L. (1971). Twenty-four hour pattern of the episodic secretion of cortisol in normal subjects. The Journal of Clinical Endocrinology and Metabolism, 33, 14-22. doi:10.1210/jcem-33-1-14.

Wemm, S., Fanean, A., Baker, A., Blough, E. R., Mewaldt, S. y Bardi, M. (2013). Problematic drinking and physiological responses among female college students. Alcohol, 47, 149-157. doi:10.1016/j.alcohol.2012.12.006.

Yamanaka, Y., Motoshima, H. y Uchida, K. (2019). Hypothalamic-pituitary-adrenal axis differentially responses to morning and evening psychological stress in healthy subjects. Neuropsychopharmacology Reports, 39, 41-47. doi:10.1002/npr2.12042.

Zimmermann, U., Spring, K., Kunz-Ebrecht, S. R., Uhr, M., Wittchen, H. U. y Holsboer, F. (2004). Effect of ethanol on hypothalamic-pituitary-adrenal system response to psychosocial stress in sons of alcohol-dependent fathers. Neuropsychopharmacology, 29, 1156-1165. doi:10.1038/ sj.npp.1300395. 